\title{
MULTI-INLET/OUTLET PRECONCENTRATOR WITH 3-D $\mu$-STRUCTURES COATED BY INKJET PRINTING OF TENAX TA
}

\author{
B. Alfeeli ${ }^{l, 2}$, M. Ashraf-Khorassani ${ }^{l}$, L. T. Taylor $^{l}$, and M. Agah ${ }^{l}$ \\ ${ }^{1}$ Virginia Polytechnic Institute and State University, Blacksburg, Virginia, USA \\ ${ }^{2}$ Kuwait Institute for Scientific Research, Kuwait City, Kuwait
}

\begin{abstract}
The design, simulation, fabrication and testing of a thermally desorbed micro preconcentrator $(\mu$-TPC) is presented. The device consists of multiple inlets/outlets with novel high-aspect-ratio $(240 \mu \mathrm{m})$ crisscross $\mu$-pillar structures and on-chip thermal desorption capability. The $\mu$-TPC has a total inner surface area of $200 \mathrm{~mm}^{2}$ and a total inner volume of $6.5 \mu \mathrm{L}$ resulting in a surface to volume ratio of $31 \mathrm{~mm}^{-1}$. The $3-\mathrm{D}$ structures were coated with Tenax TA based on polymer inkjet printing methods. The $7 \mathrm{~mm} \times$ $7 \mathrm{~mm} \mu$-TPC was capable of collecting volatile organic compounds (VOCs) with a preconcentration factor $>1000$.
\end{abstract}

\section{INTRODUCTION}

Sample pre-treatment methods, such as preconcentration, are used to increase the detection sensitivity and selectivity of many analytical instruments such as gas chromatography, mass spectrometry, and ion mobility spectrometry. Preconcentration is very useful as it aids in the detection of trace compounds such as pollutants, explosives, and drugs. The key feature of a preconcentrator is to collect an analyte or a group of analytes by adsorption over a period of time and to release them on command by thermal desorption. Conventional thermally desorbed preconcentrators (TPCs) consist of a short-length microbore tubing packed with an adsorbent material and a heating element coiled around the tube for thermal desorption. However, they are troubled by large pressure drops, dead volumes, and high power consumptions. Different configurations for MEMS-based TPCs have been reported to overcome these issues [1-4]. The development of $\mu$-TCPs enables small, sensitive, and portable analytical instruments for field measurements.

This paper reports, for the first time, a $\mu$-TCP consisting of multiple inlets/outlets with novel crisscross $\mu$-pillar structures and on-chip heaters and temperature sensors. An Optical image of the fabricated device and scanning electron microscopy (SEM) monographs of the inner structure are shown in Fig. 1. The device was placed in front of a U.S. penny to give a size prospective. The

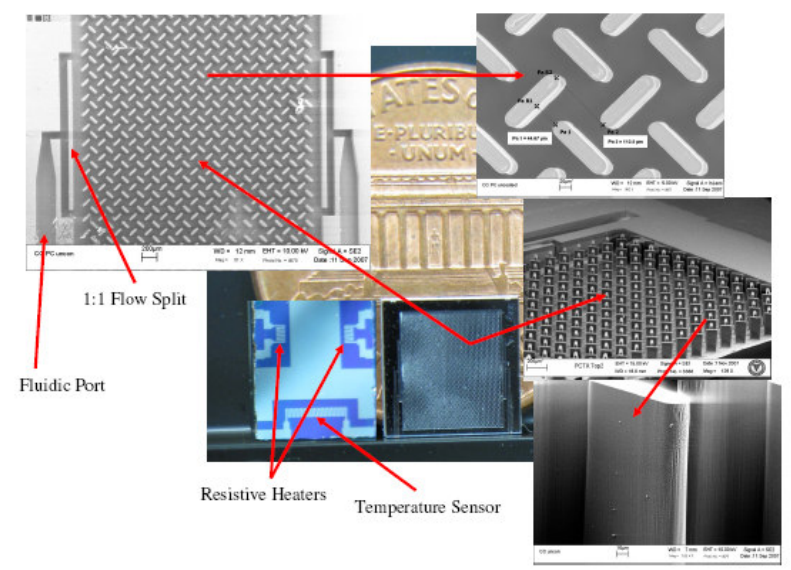

Fig. 1 Optical image of the $\mu$-TPC showing the front and back sides of the device, insets are SEM monographs of the etched 3-D structures and the fluidic ports heaters and temperature sensors are evident on the backside of the chip. The metal layer covering the rest of the surface enhances heat distribution over the entire device. The SEM monographs shows the shape and arrangement of the micropillars. The fluidic ports act as an adapter to connect the capillary tubing to the device for sample delivery and transfer. The multiple inlets/outlets were achieves by splitting the flow into a 1:1 ratio as shown in the Fig 1 . Since conventional particulate adsorbents are not suitable for this structure, a new method based on polymer inkjet printing principle [5] has been also developed to deposit Tenax TA (2,6-diphenylene oxide) on the micropillars.

\section{DESIGN AND FABRICATION}

The most important figure of merit for TPCs is the preconcentration factor (PF). It is the ratio of an analyte's concentration delivered to the detector after preconcentration to the concentration originally present in the sample. Since preconcentration relays on adsorption/desorption processes, PF is mainly dependent on the adsorption surface area. Conventional TPCs are packed with granular adsorbents. However, one effective method to enhance PF without significant pressure drops is to utilize embedded pillars and realize a hybrid configuration combining packed (large surface area) and wall-coated (small flow restrictions) structures [4].

Using computational fluid dynamics, the effectiveness of different micropillar structures was simulated in terms of flow uniformity by defining a two dimensional geometry of the device. The steady state flow through the crisscross and square pillars (for comparison) are shown in Fig 2(a-b). In the square design, the flow in the perpendicular direction cannot pass to the other side due to the relatively fast flow in the parallel direction. This forces

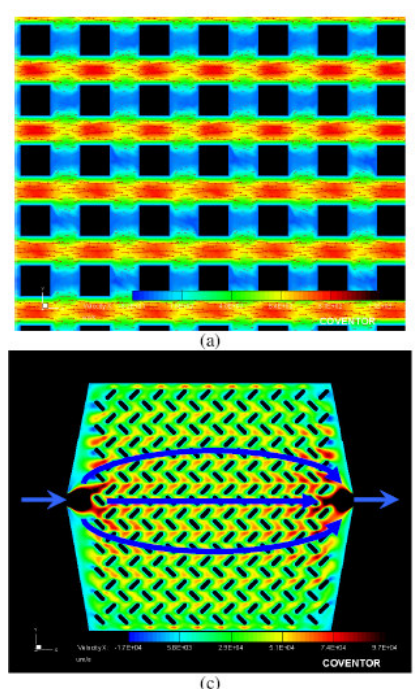

(c)
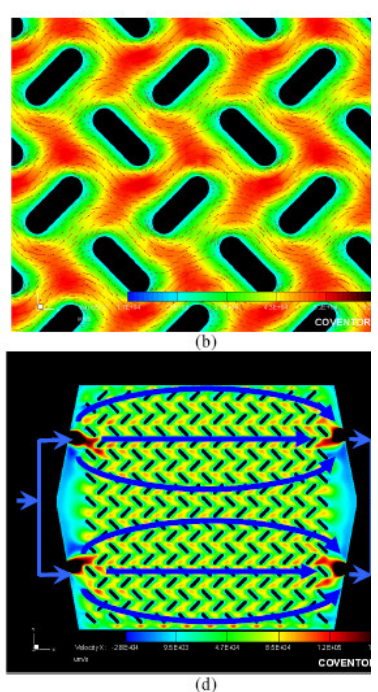

Fig. 2 CFD simulation of the flow in (a) ordered square pillars, (b) crisscross pillars, (c) crisscross pillars with single inlet/outlet showing the flow path, (d) crisscross pillars with multiple inlet/outlet showing the flow path 
the fluid to come to rest at that region creating a stagnation point as depicted by the blue region in Fig. 2(a). The sharp corners of the square pillars also contribute to stagnation. This flow pattern indicated that the sample molecules do not effectively collide with half of the pillar surfaces, thus decreasing the adsorption surface. In the crisscross design, the sharp corners were eliminated and the flow was directed in a zigzag pattern to ensure non-zero velocity in all direction. The simulation analysis also showed that the multiple inlet/outlet design spreads the flow more evenly among the pillars (Fig. 2c-d). In the single inlet/outlet design, the flow velocity was highest in the middle giving rise to an undesired flow concentration. The use of a double inlet/outlet lessened this effect allowing more interaction between the pillars and the gaseous sample.

Fig. 3 shows the fabrication process. Using the standard photolithographic technique, the micropillars and the fluidic ports were patterned on a 4" silicon wafer. Deep reactive ion etching (DRIE) was utilized to form the $240 \mu \mathrm{m}$-deep pillars and fluidic

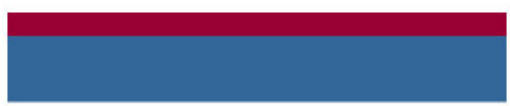

(a)

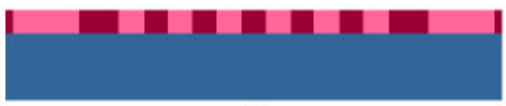

(b)

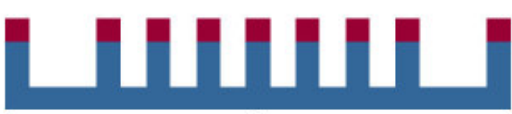

(c)

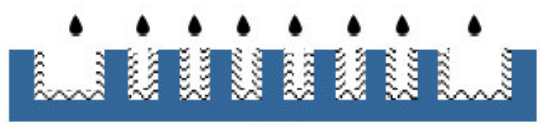

(d)

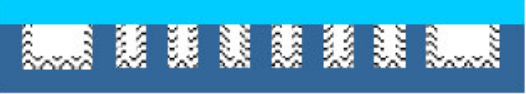

(e)

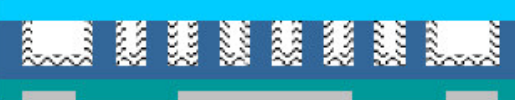

(f)

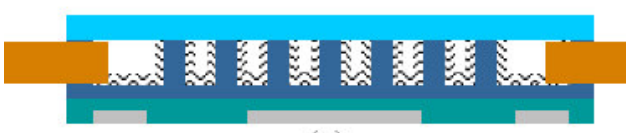

(g)

Fig. 3 (a) Spinning of high-resolution, superior aspect ratio thick film photoresist (PR9260), (b) Photolithography of $\mu$ pillars and the fluidic ports, (c) The pattern was etched using deep reactive-ion etching (DRIE) to form $240 \mu \mathrm{m}$ deep 3D structures, (d) Dispensing of one-by-one droplets of adsorbent polymer solution on the microstructures, (e) Anodic bonding with Pyrex glass wafer to seal the structures, (f) PECVD oxidation followed by the deposition of thin-film resistive heaters and temperature sensors, $(\mathrm{g})$ Attaching deactivated fused silica tubing to the fluidic ports by high temperature silica based bonding agent. ports. Tenax TA particles were dissolved in dichloromethane at 2 $\mathrm{mg} / \mathrm{mL}$ concentration to create a solution for inkjet printing. Before bonding, $\mu$-droplets were dispensed one-by-one on the 3-D microstructures. The volatility of the solvent and the large surface to volume ratio of the $\mu$-droplets caused instant evaporation which left behind a film of Tenax TA on the surface. This method resulted in a uniform and high-coverage coating as demonstrated in Fig. 4. The $\mu$-TPC was then sealed by anodic bonding the silicon substrate to a Pyrex wafer at $1250 \mathrm{~V}$ and $250^{\circ} \mathrm{C}$ instead of conventional $350-400^{\circ} \mathrm{C}$ bonding temperature to avoid damaging the polymer. After depositing a $3000 \AA$ PECVD oxide layer on the backside, Ti/Pt was evaporated and patterned to form resistive heaters and temperature sensors.

\section{EXPERIMENT SETUP}

To maintain isothermal temperature condition, the testing was carried out inside a commercial GC oven. The built-in auxiliary systems such as gas flow controller and flame ionization detector (FID) were also utilized to ensure consistent testing parameters. Helium carrier gas was supplied via the GC inlet and controlled by the flow controller. The sample adsorption/desorption was measured by the FID. The on-board resistive heaters and temperature sensor were not used in this experiment. Instead, an off-board high-performance ceramic heater (ultra-fast ramp rate) was used for thermal desorption along with K-type thermocouple for manual temperature monitoring and control. In future work, the on-chip thermal desorption capability will be used.

The experimental testing was performed by loading the chip with pure n-nonane $\left(\mathrm{C}_{9}\right)$ and then thermally desorbing it at $100^{\circ} \mathrm{C} / \mathrm{sec}$ up to $250^{\circ} \mathrm{C}$. This was done for uncoated devices with
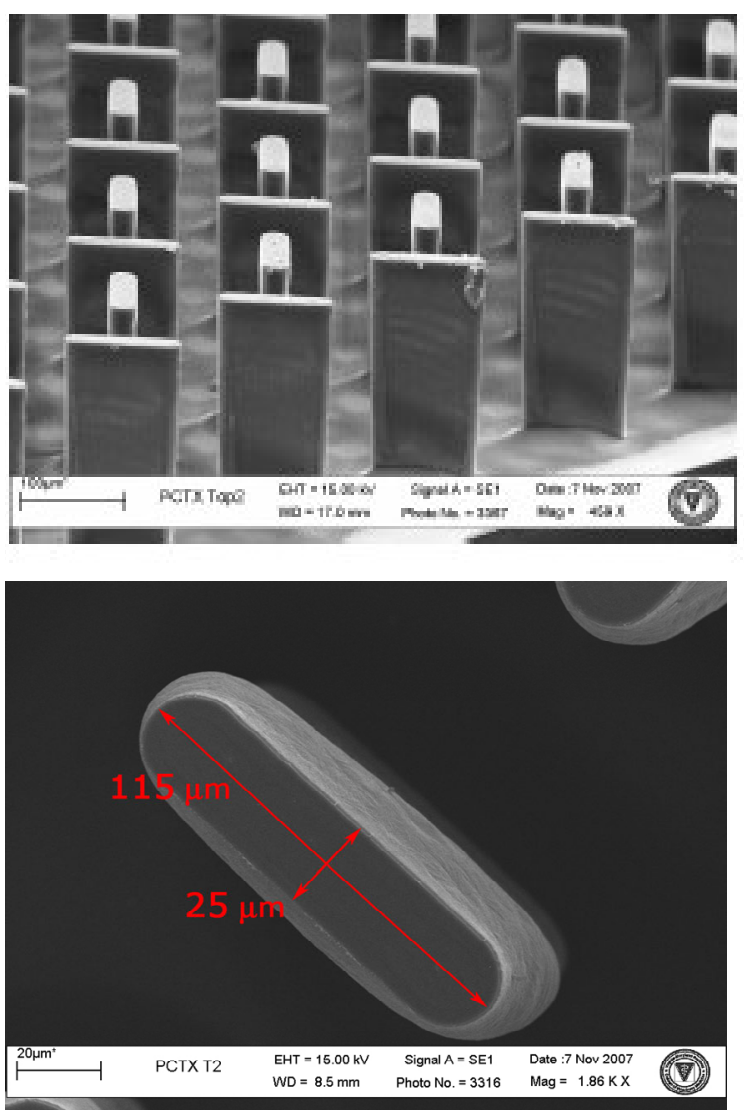

Fig. 4 SEM monographs of Tenax TA coated pillars. 
the following configurations: without pillars, with square pillars, and crisscross pillars. This initial test was carried out to confirm the simulation results. All subsequent tests were performed on the crisscross design coated with Tenax TA since it was the focus of this work.

To examine the use of the $\mu$-TPC as an injector, the device was put on-line with a $2 \mathrm{~m}$-long, $100 \mu \mathrm{m}$-ID fused silica capillary column coated in-house with a methyl polysiloxane stationary phase. The $\mu$-TPC was then loaded with a diluted mixture of $n-$ octane $\left(\mathrm{C}_{8}\right)$, n-nonane $\mathrm{C}_{9}$, n-decane $\left(\mathrm{C}_{10}\right)$, n-dodecane $\left(\mathrm{C}_{12}\right)$, and $\mathrm{n}$ tetradecane $\left(\mathrm{C}_{14}\right)$. The injection of the mixture into the $\mathrm{GC}$ column was initiated by the fast thermal desorption. The column ramp was $10^{\circ} \mathrm{C} / \mathrm{min}$ from $35^{\circ} \mathrm{C}$ to $150^{\circ} \mathrm{C}$.

\section{RESULTS AND DISCUSSION}

Using the aforementioned fabrication process, $\mu$-TPC devices with high-aspect-ratio structures $(240 \mu \mathrm{m})$ were developed on a 7 $\mathrm{mm}$ square die. The $\mu$-TPC with crisscross pillars has a total inner surface area of about $200 \mathrm{~mm}^{2}$ and a total inner volume of about $6.5 \mu \mathrm{L}$ resulting in $\sim 31 \mathrm{~mm}^{-1}$ surface area to volume ratio $(\mathrm{S} / \mathrm{V})$. For comparison, a $25 \mathrm{~cm}$-long, $250 \mu \mathrm{m}$-ID capillary tube yields the same surface area but has an inner volume of $12.5 \mu \mathrm{L}$, and $\mathrm{S} / \mathrm{V}$ of $16 \mathrm{~mm}^{-1}$.

The effect of the pillar design and configuration as well as the number of inlet/outlet on the $\mu$-TPC performance was studied by testing the performance of the uncoated and coated devices. Testing uncoated devices enabled performance characterization without interference effects from the adsorbent material. Table 1 lists the preconcentration factor for no-pillars, square and crisscross pillars along with single and multiple inlet/outlet crisscross devices. As predicted by the simulation analysis, the double inlet/outlet crisscross device produced the highest concentration factor among the other designs. The elimination of

Table 1: Preconcentration factor for different designs

\begin{tabular}{|c|c|c|c|}
\hline Design & inlet/outlet & $\begin{array}{c}\text { Spacing between } \\
\text { posts }(\boldsymbol{\mu} \text { m) }\end{array}$ & PF factor \\
\hline No posts & 1 & - & 4 \\
\hline Ordered & 1 & 50 & 7 \\
\hline Crisscross & 1 & 80 & 22 \\
\hline Crisscross & 2 & 80 & 32 \\
\hline Crisscross & 1 & $80+$ Tenax TA & 700 \\
\hline Crisscross & & & \\
\hline
\end{tabular}

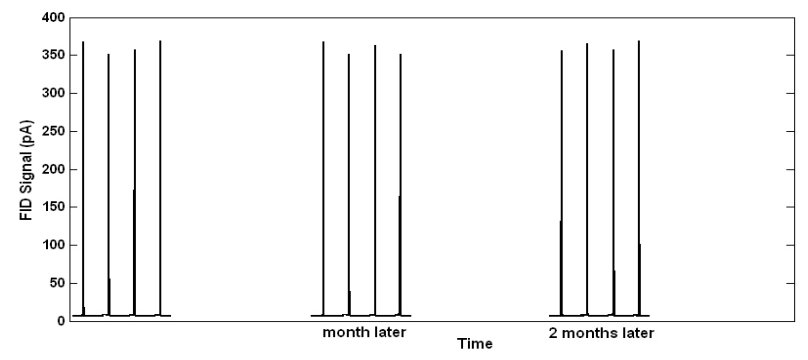

Fig. 5 Series of $\mu$-TPC generated desorption pulses over a three-month period.

stagnation and the effective dispersing of the sample among pillars produced more vapor/surface interaction. Moreover, the double inlet/outlet splits the flow into two flows with half the magnitude of the incoming flow (See Fig. 2 (d)). This led to a slower flow within the device compared to the flow in the single inlet/outlet configuration making the adsorption process more effective.

Fig. 5 demonstrates a series of desorption pulses generated over three months by a coated $\mu$-TPC consisting crisscross pillars with double inlet/outlet configuration. Reproducibility in terms of peak height and width was excellent (relative standard deviation $3 \%$ and $6 \%$ respectively).

Fig. $6 \mathrm{a}$ and $6 \mathrm{~b}$ represent the chromatogram obtained by injecting the sample through the GC conventional injector and the $\mu$-TPC, respectively. The fast thermal desorption enabled sharp peak injection comparable to what could be produced by the GC injection port. The solvent peak was significantly smaller in the $\mu$-TPC injection pulse indicating that the device did not concentrate most of the solvent (dichloromethane). Yet, most of the separated compounds show a higher peak when injected through the $\mu$-TPC as clearly demonstrated in the zoomed-in chromatograms. Comparing the peak magnitudes of the solvent shows that the $\mu$-TPC has the advantage of discriminating between VOCs and the solvent. Therefore, the device can be used as a split injector to reduce the amount of solvent entering the GC column. The slight shift in the elution time of compounds is attributed to the change in the flow due to the placement of the $\mu$-TPC.

\section{CONCLUSIONS}

The sensitivity and selectivity of an analytical instrument could be improved with preconcentration as a sample pretreatment. This report has demonstrated the possibility of developing MEMSbased $\mu$-TPCs that have smaller sizes, lower pressure drops, lower power consumptions, and higher efficiencies compared to conventional counterparts. By utilizing novel crisscross pillars with zigzag configuration and multiple inlet/outlet design, we have demonstrated a hybrid configuration combining packed (large surface area) and wall-coated (small flow restrictions) structures.

The simulation analyses verified by experimental results have shown the effect of the distributed inlet/outlet as well as the pillar geometry and configuration on the $\mu$-TPC performance. Future work will aim to better understand and model the behavior of the $\mu$-TPC under different conditions

The Sharp reproducible desorption peaks needed for fast onfield analytical analysis has been demonstrated by the $\mu$-TPC. The presented device was successful in concentrating a diluted multicompound hydrocarbon mixture. 

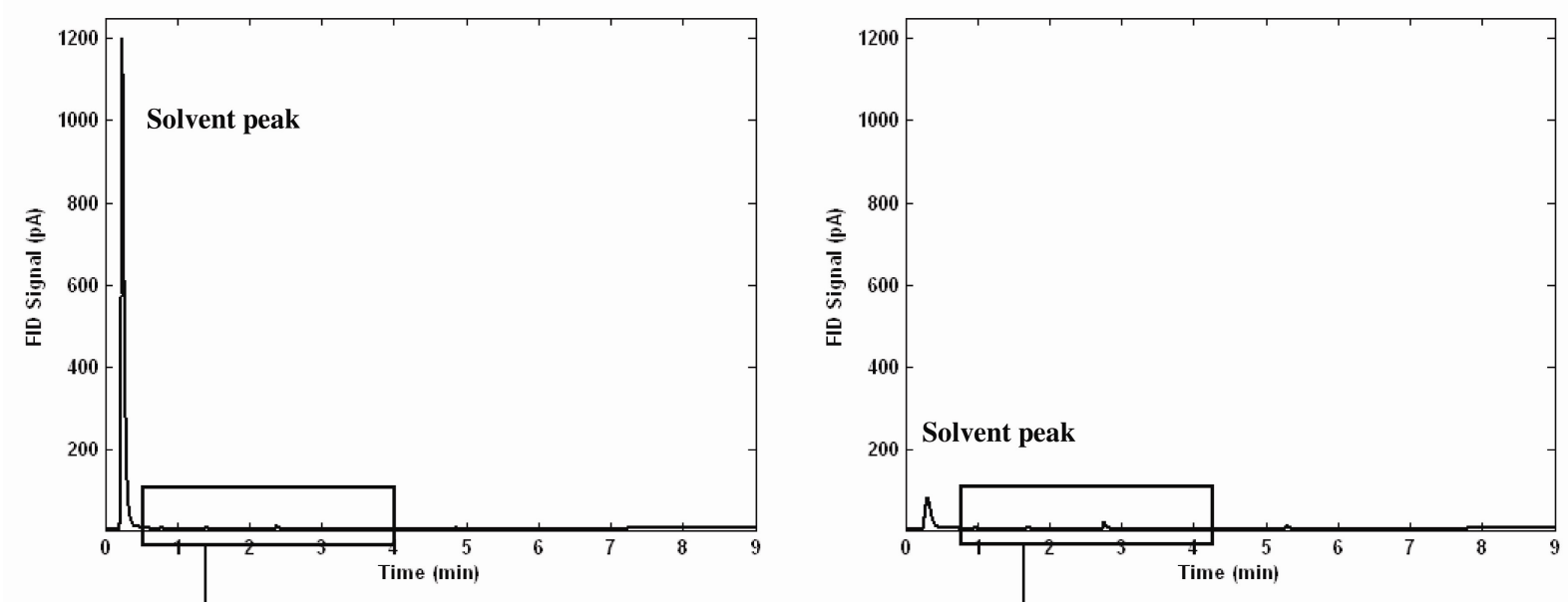

(a)

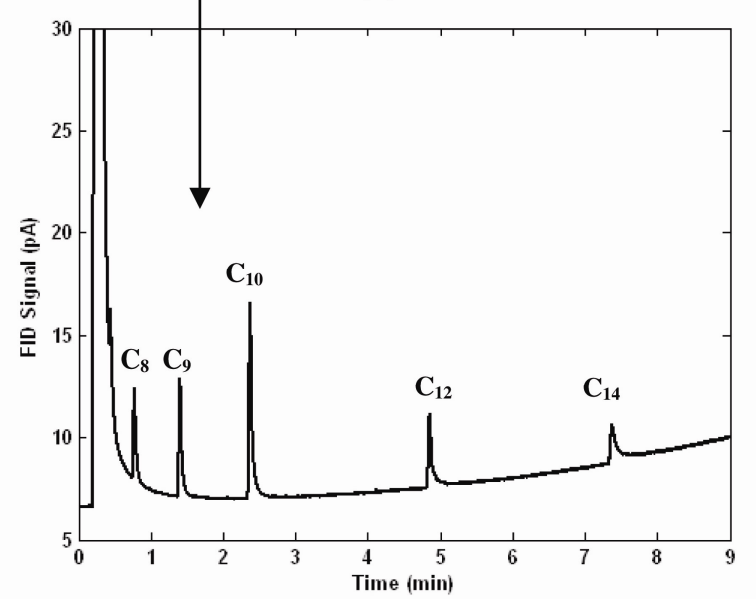

(b)

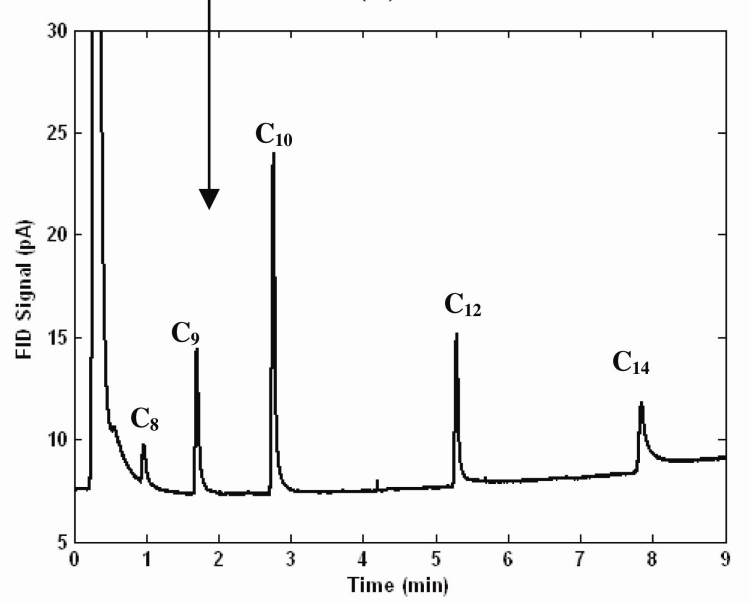

Fig. 6 Sample injection chromatograms, (a) represents the sample injected through the GC conventional injection port with a zoomed-in version below it, and $(b)$ represents the sample injected by the $\mu$-TPC fast thermal desorption ability with a zoomed-in version below it

\section{REFERENCES}

[1] P. R. Lewis, P. Manginell, D. R. Adkins, R. J. Kottenstette, D. R. Wheeler, S. S. Sokolowski, D. E. Trudell, J. E. Byrnes, M. Okandan, J. M. Bauer, R. G. Manley and C. Frye-Mason, "Recent advancements in the gas-phase MicroChemLab," IEEE Sens. J. 6, 3, (2006)

[2] W.-C. Tian, H. K. L. Chan, L. Chia-Jung, S. W. Pang and E. T. Zellers, "Multiple-stage microfabricated preconcentratorfocuser for micro gas chromatography system," $J$. Microelectromech. Syst. 14, 3, (2005)

[3] M. Kim and S. Mitra, "A microfabricated microconcentrator for sensors and gas chromatography," J. Chromatogr. A 996, $1-2,(2003)$

[4] Y. Tang, J. Yeom, J. Han, B. Bae, R. I. Masel and M. A. Shannon, "A Micro-post preconcentrator for a microscale gas chromatography system", 9th International Conference on Miniaturized Systems for Chemistry and Life Sciences, Boston, MA, (2005), 660-662

[5] P. Calvert, "Inkjet Printing for Materials and Devices," Chem. Mater. 13, 10, (2001) 